Research Article

\title{
Quantitative Determination of $p$-Cymene, Thymol, Neryl Acetate, and $\beta$-Caryophyllene in Different Growth Periods and Parts of Eupatorium fortunei Turcz. by GC-MS/MS
}

\author{
Guanjun Nan ${ }^{D},{ }^{1}$ Lina Zhang, ${ }^{1}$ Zhengzheng Liu, ${ }^{1}$ Yu Liu, ${ }^{1}$ Yan Du, ${ }^{1}$ Hongwen Zhao, \\ Hongxia Zheng, ${ }^{1}$ Rong Lin, ${ }^{2}$ Guangde Yang $(1),{ }^{1}$ and Shaohua Zheng $\mathbb{1}^{3}$ \\ ${ }^{1}$ School of Pharmacy, Xi'an Jiaotong University, Xi'an 710061, China \\ ${ }^{2}$ Department of Pharmacology, Xi'an Jiaotong University, Xi'an 710061, China \\ ${ }^{3}$ Department of Anesthesiology and Operation, The First Affiliated Hospital of Xi'an Jiaotong University, Xi'an 710061, China
}

Correspondence should be addressed to Guangde Yang; jmw52@xjtu.edu.cn and Shaohua Zheng; zhengsh@xjtu.edu.cn

Received 21 April 2021; Revised 2 July 2021; Accepted 12 July 2021; Published 2 August 2021

Academic Editor: Giuseppe Ruberto

Copyright (C) 2021 Guanjun Nan et al. This is an open access article distributed under the Creative Commons Attribution License, which permits unrestricted use, distribution, and reproduction in any medium, provided the original work is properly cited.

Eupatorium fortunei Turcz. is a widely used Chinese herbal medicine in China. In this study, a gas chromatography-triple quadrupole mass spectrometry (GC-MS/MS) method was developed and validated to simultaneously determine the contents of $p$ cymene, thymol, neryl acetate, and $\beta$-caryophyllene in roots, stems, and leaves of Eupatorium fortunei Turcz. harvested at different growth periods. All four constituents could be detected in leaves, three could be detected in stems except $\beta$-caryophyllene, and only thymol could be detected in roots. The order of the total contents of four constituents in different parts was leaves $>$ stems $>$ roots. It indicated that the leaves could be the proper medicinal parts of Eupatorium fortunei Turcz. The content of four constituents in leaves varied a lot among different growth periods and showed an M-shaped change trend with the growth of Eupatorium fortunei Turcz. The four constituents accumulated to the highest values in early July followed by mid-September. Accordingly, the best harvest time of Eupatorium fortunei Turcz. is early July and mid-September.

\section{Introduction}

Eupatorium fortunei Turcz., one species of the genus Eupatorium (Compositae), is a commonly used aromatic traditional herbal medicine in China. It has been applied for relieving nausea, vomiting, and poor appetite due to dampness obstruction since thousands of years ago [1]. Previous studies showed that the plant contains a number of bioactive natural products $[2,3]$. The content determination of total volatile oil in Eupatorium fortunei Turcz. is stipulated in Chinese Pharmacopeia, and the total volatile oil is consequently used as an important index to evaluate its quality [4], while various studies showed that constituents in the volatile oil of Eupatorium fortunei Turcz. from different regions and harvest time varied a lot [5-7]. Accordingly, it is of great significance to assay representative constituents in the volatile oil of Eupatorium fortunei Turcz.

Based on the related literature, $p$-cymene and neryl acetate were the earliest separated and identified compounds from the volatile oil of Eupatorium fortunei Turcz. [8]. It has been reported that $p$-cymene possesses an effect on dispelling phlegm [9]. Together with neryl acetate, it was also claimed to be the main active ingredient of the volatile oil for the treatment of influenza [10]. $\beta$-Caryophyllene was reported to be the constituent with the highest content in the volatile oil and has been studied as an indicator constituent to establish the content determination method of Eupatorium fortunei Turcz. $[11,12]$. Thymol and its derivatives are abundant in the plant $[2,13-15]$. It could be concluded that the contents of $p$-cymene, thymol, neryl acetate, and 
$\beta$-caryophyllene (structured as shown in Figure 1) have a significant effect on the quality of Eupatorium fortunei Turcz.

It is well known that traditional Chinese medicines have the characteristic of complex composition. The composition and content of compounds are closely related to the quality of traditional Chinese medicine. The growth period is always an important factor affecting the composition and content of constituents in the medicinal herbs. Thus, the appropriate harvesting time for traditional Chinese medicine is crucial to the formation of high-quality traditional Chinese medicine [16]. A lot of works have been performed on the effect of the developmental stage on the content of biologically active compounds in plants [17-19]. Accordingly, the quantities of the volatile oil in Eupatorium fortunei Turcz. may change during the growth stages. It is essential to study the content change of the volatile oil with growth for ensuring the quality of Eupatorium fortunei Turcz. to prevent and treat disease.

Therefore, in this work, Eupatorium fortunei Turcz. was harvested at different growth periods, and the contents of $p$ cymene, thymol, neryl acetate, and $\beta$-caryophyllene in different parts were determined by using GC-MS/MS. The dynamic characteristic and distribution of four constituents were analyzed.

\section{Materials and Methods}

2.1. Plant Materials. Eupatorium fortunei Turcz. was collected every fifteen days from April 15 to November 1, 2019, at the medicinal botanical garden of Xi' an $\left(34^{\circ} 25^{\prime} \mathrm{N}, 108^{\circ} 98^{\prime}\right.$ E) and identified by Prof. Xiaofeng Niu. Ten plants were harvested at each stage. Each plant was separated into leaves, stems, and roots. Then, the leaves, stems, and roots were separately collected together and dried.

2.2. Reagents and Chemicals. Ethanol, ethyl acetate, petroleum ether $\left(60^{\circ} \mathrm{C} \sim 90^{\circ} \mathrm{C}\right)$, and hexane were of analytical grade. $p$-Cymene (purity: 99.5\%) and neryl acetate (purity: 95\%) were purchased from Shanghai Aladdin BioChem Technology Co., Ltd. (Shanghai, China). Thymol (purity: 99\%) and $\beta$-caryophyllene (purity: 90\%) were purchased from J\&K Scientific Ltd. (Beijing, China).

2.3. GC-MS/MS Analysis. A Shimadzu GC-MS 8040 equipped with SH-Rxi-5Sil MS capillary column $(30 \mathrm{~m}$, $0.25 \mathrm{~mm}$ i.d., $0.25 \mu \mathrm{m}$ film thickness) and triple quadrupole mass spectrometer was used (Japan). The helium of high purity (99.9999\%) was the carrier gas at a flow rate of $1 \mathrm{~mL} \cdot \mathrm{min}^{-1}$. Samples were injected in a split mode with a ratio of $10: 1$. The temperature procedure of the column was set at $70^{\circ} \mathrm{C}$ for $3 \mathrm{~min}$, increased to $80^{\circ} \mathrm{C}$ at a rate of $10^{\circ} \mathrm{C} \cdot \mathrm{min}^{-1}$, to $100^{\circ} \mathrm{C}$ at a rate of $20^{\circ} \mathrm{C} \cdot \mathrm{min}^{-1}$, to $130^{\circ} \mathrm{C}$ at a rate of $2^{\circ} \mathrm{C} \cdot \mathrm{min}^{-1}$, and to $280^{\circ} \mathrm{C}$ at a rate of $20^{\circ} \mathrm{C} \cdot \mathrm{min}^{-1}$, and maintained for $2 \mathrm{~min}$. An electron ionization system was used with ionization energy of $70 \mathrm{eV}$. Injection, interface, and ionization temperature were set at $250^{\circ} \mathrm{C}, 250^{\circ} \mathrm{C}$, and $230^{\circ} \mathrm{C}$, respectively. We used standard compounds of each volatile under the MRM mode to assay the contents of four target components [20].

2.4. Preparation of Standard Solution and Samples. Each standard stock solution was prepared separately by dissolving accurate amount of compound in hexane. A series of working solutions of these 4 compounds were freshly prepared by diluting the mixed standard solution with hexane.

Dried leaves, stems, and roots of Eupatorium fortunei Turcz. were milled to powder by using a high-speed disintegrator, and $0.2 \mathrm{~g}$ ground powder was extracted with $20 \mathrm{~mL}$ hexane in an ultrasonic waterbath for $60 \mathrm{~min}$. Additional hexane was added to make up the lost weight [16]. Then, the solution was filtered through a $0.22 \mu \mathrm{m}$ filter before GC-MS/MS analysis.

2.5. Statistical Analysis. All measurements were performed using triplicate samples. Data were expressed as mean \pm SD. All data were statistically processed using GraphPad Prism 7 (GraphPad Software, CA, USA) and analyzed by Tukey's multiple comparisons test to calculate the difference significance at a $5 \%$ level $(p<0.05)$.

\section{Results and Discussion}

3.1. Optimization of GC-MS Analysis Conditions and Method Validation. Temperature procedure, parameters of ion pairs, and collision voltage are the key parameters for the GC-MS/MS method. These parameters were optimized in the present study. MRM was used for quantitative purposes with one target ion pair and two reference ion pairs. The corresponding collision voltage was optimized and is listed in Table 1. The MRM chromatogram is shown in Figure 2.

Linearity, range, accuracy, precision, limits of detection (LOD), and limits of quantification (LOQ) were evaluated under the optimal conditions (Table 2). p-Cymene, thymol, neryl acetate, and $\beta$-caryophyllene could be determined in a linear range of $0.01 \mu \mathrm{g} \cdot \mathrm{mL}^{-1} \sim 0.38 \mu \mathrm{g} \cdot \mathrm{mL}^{-1}$, $0.05 \mu \mathrm{g} \cdot \mathrm{mL}^{-1} \sim 6.21 \mu \mathrm{g} \cdot \mathrm{mL}^{-1}, \quad 0.11 \mu \mathrm{g} \cdot \mathrm{mL}^{-1} \sim 0.88 \mu \mathrm{g} \cdot \mathrm{mL}^{-1}$, and $0.13 \mu \mathrm{g} \cdot \mathrm{mL}^{-1} \sim 2.19 \mu \mathrm{g} \cdot \mathrm{mL}^{-1}$ with good correlation coefficients $(r>0.999)$, respectively. LODs and LOQs were calculated at a signal-to-noise of 3 and 10. Accuracy was measured by recoveries. The percentage recoveries were $105.31 \%, 106.41 \%, 99.61 \%$, and $102.24 \%$ for $p$-cymene, thymol, neryl acetate, and $\beta$-caryophyllene, respectively. The repeatability of the method was determined by analyzing the standard solution 6 times. The relative standard deviations (RSD) for $p$-cymene, thymol, neryl acetate, and $\beta$-caryophyllene were $1.00 \%, 2.72 \%, 1.55 \%$, and $1.59 \%$, respectively. Six homogeneous samples were prepared and analyzed to evaluate the precisions. The RSD values of four analytes varied from $0.98 \%$ to $3.38 \%$. For stability, the same sample was assayed separately at $0,2,4,6,8$, and $12 \mathrm{~h}$ after extraction. The RSD values were less than $1.36 \%$.

The results demonstrated that the developed method was accurate and repeatable which could be a reliable tool for identification and quantification of $p$-cymene, thymol, neryl acetate, and $\beta$-caryophyllene in Eupatorium fortunei Turcz. 


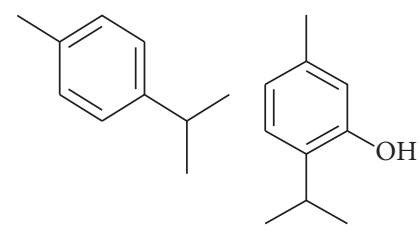

(a)

(b)

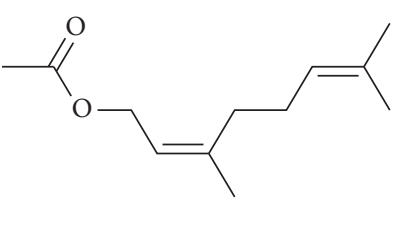

(c)

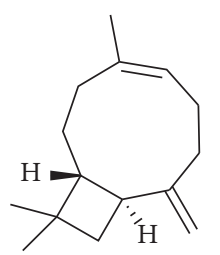

(d)

Figure 1: Chemical structures of (a) p-cymene, (b) thymol, (c) neryl acetate, and (d) $\beta$-caryophyllene.

TABLE 1: Target ion pair, reference ion pairs, and collision voltage of compounds.

\begin{tabular}{lcccccc}
\hline Compounds & $\begin{array}{c}\text { Target ions }(\mathrm{m} / \\
\mathrm{z})\end{array}$ & $\begin{array}{c}\text { Collision voltage } \\
(\mathrm{eV})\end{array}$ & $\begin{array}{c}\text { Reference ions 1 } \\
(\mathrm{m} / \mathrm{z})\end{array}$ & $\begin{array}{c}\text { Collision voltage } \\
(\mathrm{eV})\end{array}$ & $\begin{array}{c}\text { Reference ions 2 } \\
(\mathrm{m} / \mathrm{z})\end{array}$ & $\begin{array}{c}\text { Collision voltage } \\
(\mathrm{eV})\end{array}$ \\
\hline p-Cymene & $119>91.1$ & 15 & $134>119.1$ & 9 & $119>117.1$ & 12 \\
Thymol & $135>91.1$ & 18 & $150>135.1$ & 9 & $135>115.1$ & 15 \\
Neryl acetate & $93>77.1$ & 12 & $93>91.1$ & 12 & $93>51.1$ & $91>65.1$ \\
$\beta$-Caryophyllene & $93>77.1$ & 12 & $93>91.1$ & 9 & 24 \\
\hline
\end{tabular}

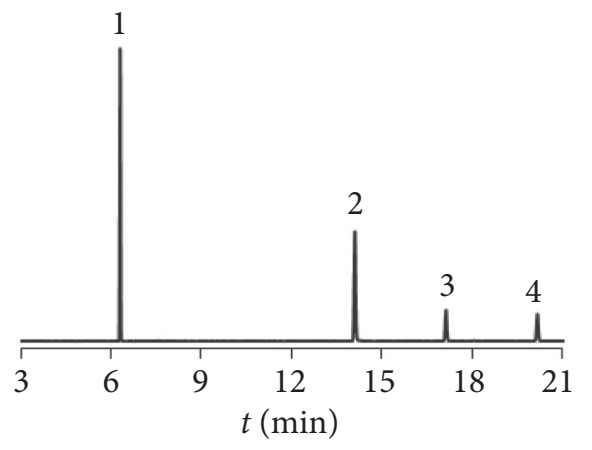

(a)

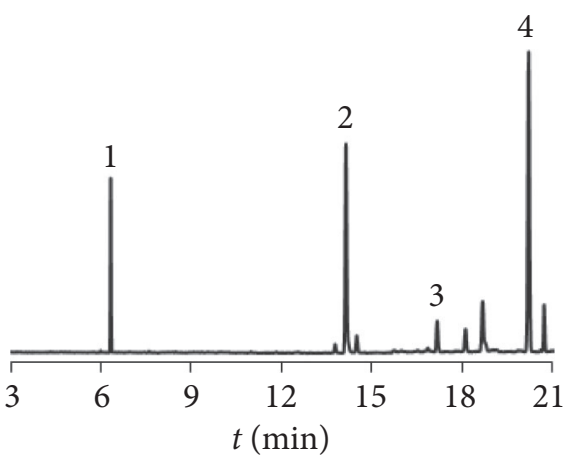

(b)

Figure 2: The MRM chromatogram of (a) references and (b) samples (1, p-cymene; 2 , thymol; 3 , neryl acetate; 4 , $\beta$-caryophyllene).

TABLe 2: Validation of the optimized GC-MS/MS.

\begin{tabular}{lcccccccc}
\hline Compounds & $\begin{array}{c}\text { Regression } \\
\text { equation }\end{array}$ & $r$ & $\begin{array}{c}\text { LOD } \\
\left(\mathrm{ng} \cdot \mathrm{mL}^{-1}\right)\end{array}$ & $\begin{array}{c}\text { LOQ } \\
\left(\mathrm{ng} \cdot \mathrm{mL}^{-1}\right)\end{array}$ & $\begin{array}{c}\text { Precision } \\
(\mathrm{RSD} \%)\end{array}$ & $\begin{array}{c}\text { Recovery } \\
(\%)\end{array}$ & $\begin{array}{c}\text { Repeatability } \\
(\mathrm{RSD} \%)\end{array}$ & $\begin{array}{c}\text { Stability } \\
(\mathrm{RSD} \%)\end{array}$ \\
\hline$p$-Cymene & $y=33388 x-46.065$ & 0.9995 & 6 & 12 & 3.38 & 105.31 & 1.00 \\
Thymol & $y=19378 x-523.59$ & 0.9996 & 14 & 54 & 2.98 & 106.41 & 1.11 \\
Neryl acetate & $y=8149 x-441.05$ & 0.9990 & 28 & 110 & 0.98 & 99.61 & 1.72 \\
$\beta$-Caryophyllene & $y=8152 x-327.55$ & 0.9992 & 38 & 129 & 2.70 & 102.24 & 1.55 \\
\hline
\end{tabular}

3.2. Optimization of Sample Process Conditions. The influence factors, including extraction solvent, method, and duration were investigated through single-factor experiments. Extraction solvents including ethanol, ethyl acetate, petroleum ether $\left(60^{\circ} \mathrm{C} \sim 90^{\circ} \mathrm{C}\right)$, and hexane were compared, and hexane resulted in a preferable peak and baseline. Two classical extraction methods, ultrasonic extraction, and reflux, were investigated. The ultrasonic extraction showed better extraction efficiency. Then, we set the extraction duration at $15 \mathrm{~min}, 30 \mathrm{~min}, 45 \mathrm{~min}$, and $60 \mathrm{~min}$. The contents of compounds were increased with the prolongation of extraction time (Table 3 ). Therefore, the extraction was conducted by using hexane in an ultrasonic waterbath for $60 \mathrm{~min}$. 
TABLE 3: Investigation of extraction conditions.

\begin{tabular}{lcccr}
\hline & $p$-Cymene & Thymol & Neryl acetate & $\beta$-Caryophyllene \\
\hline Extraction method $\left(\mu \mathrm{g} \cdot \mathrm{g}^{-1}\right)$ & & & & \\
$\quad$ Ultrasonic & $3.67 \pm 0.079$ & $96.98 \pm 0.759$ & $47.49 \pm 1.390$ & $58.70 \pm 0.642$ \\
$\quad$ Reflux & $2.96 \pm 0.137$ & $77.59 \pm 0.461$ & $30.82 \pm 0.227$ & $10.49 \pm 0.661$ \\
\hline Extraction duration $\left(\mu \mathrm{g} \cdot \mathrm{g}^{-1}\right)$ & & & \\
$15 \mathrm{~min}$ & $2.07 \pm 0.040 \mathrm{c}$ & $61.60 \pm 0.632 \mathrm{~d}$ & $42.22 \pm 0.651 \mathrm{~d}$ & $28.53 \pm 0.449 \mathrm{~d}$ \\
$30 \mathrm{~min}$ & $3.67 \pm 0.079 \mathrm{~b}$ & $96.98 \pm 0.759 \mathrm{c}$ & $47.49 \pm 1.390 \mathrm{c}$ & $58.70 \pm 0.642 \mathrm{c}$ \\
$45 \mathrm{~min}$ & $4.01 \pm 0.079 \mathrm{a}$ & $107.06 \pm 1.937 \mathrm{~b}$ & $60.16 \pm 0.940 \mathrm{~b}$ & $65.36 \pm 1.282 \mathrm{~b}$ \\
$60 \mathrm{~min}$ & $4.12 \pm 0.139 \mathrm{a}$ & $111.80 \pm 2.317 \mathrm{a}$ & $67.32 \pm 0.659 \mathrm{a}$ & $70.64 \pm 1.906 \mathrm{a}$ \\
\hline
\end{tabular}

Data are presented as mean \pm standard deviation. Values with the same letter within each column are not significantly different $(p<0.05)$.

3.3. Distribution of Four Constituents in Different Parts of Eupatorium fortunei Turcz. Samples from different growth periods and parts were assayed. The contents of $p$-cymene, thymol, neryl acetate, and $\beta$-caryophyllene in different parts of Eupatorium fortunei Turcz. are listed in Table 4.

The contents of $p$-cymene, thymol, neryl acetate, and $\beta$-caryophyllene in leaves were ranged from $1.51 \pm 0.056$ to $25.25 \pm 2.600 \mu \mathrm{g} \cdot \mathrm{g}^{-1}, 20.81 \pm 0.688$ to $132.21 \pm 3.169 \mu \mathrm{g} \cdot \mathrm{g}^{-1}$, $13.77 \pm 2.600$ to $59.90 \pm 1.006 \mu \mathrm{g} \cdot \mathrm{g}^{-1}$, and $13.36 \pm 2.611$ to $144.21 \pm 1.365 \mu \mathrm{g} \cdot \mathrm{g}^{-1}$, respectively. $\beta$-caryophyllene could not be detected in stems. The concentration of $p$-cymene, thymol, and neryl acetate in stems were within the range of $1.79 \pm 0.034 \sim 5.90 \pm 0.659 \mu \mathrm{g} \cdot \mathrm{g}^{-1}, 5.48 \pm 0.043 \sim 19.10 \pm 1.729$ $\mu \mathrm{g} \cdot \mathrm{g}^{-1}$, and $12.51 \pm 0.115 \sim 40.94 \pm 0.182 \mu \mathrm{g} \cdot \mathrm{g}^{-1}$, respectively. Only thymol could be detected in roots, and the contents ranged from $5.42 \pm 0.547$ to $32.60 \pm 0.409 \mu \mathrm{g} \cdot \mathrm{g}^{-1}$. In a previous study, it has been found that the compounds from the volatile oils extracted from Eupatorium fortunei Turcz. with the three methods were different [21]. The treatment and processing of herbs also affect the composition and content of volatile oil from Eupatorium fortunei Turcz. [22].

All the constituents could be detected in leaves, and only thymol exists in roots. The order of the contents of four constituents in different parts was leaves $>$ stems $>$ roots. Volatile oil exists in glandular hairs, oil rooms, oil tubes, secretory cells, or resin ducts. Volatile oil exists in different parts of different plants. Some of them are found in the whole plant, while others are found in some organs of flowers, fruits, leaves, roots, or rhizomes. The location of volatile oil varies with plant species. Hence, one can see that the studied four compounds mainly existed in leaves of Eupatorium fortunei Turcz. Higher content of essential oil in leaf than other organs of a plant has been previously reported for other species $[19,23]$. Based on that, it could be concluded that the leaves should be the best medicinal part of Eupatorium fortunei Turcz.

3.4. Dynamic Characteristics of Four Constituents in Different Growth Periods of Eupatorium fortunei Turcz. The concentrations change of $p$-cymene, thymol, neryl acetate, and $\beta$-caryophyllene in different parts of Eupatorium fortunei Turcz. with growth time were also analyzed. The contents of all constituents in leaf showed a trend of increase-decrease-increase-decrease with the plant growing. The content of $p$-cymene accumulated to its highest value on July 15 . After an $80 \%$ reduction, the content rose again to $86 \%$ of the highest value approximately on September 15. Thymol reached its first peak value on August 15 and then reached the highest level on September 15. The highest value of thymol was about 6.5 times and 3.3 times higher than that on September 1 and October 1. The content of neryl acetate was the highest from July 1 to July 15, and it was also high on September 15 , which is about $74 \%$ of the highest value. The yield of $\beta$-caryophyllene was the highest on July 1 and then decreased sharply. The content of $\beta$-caryophyllene increased again from $17.86 \mu \mathrm{g} \cdot \mathrm{g}^{-1}$ on September 1 to $44.38 \mu \mathrm{g} \cdot \mathrm{g}^{-1}$ on September 15 . The contents of four constituents in the stem at each harvest stage showed different patterns. The yield of $p$-cymene was the highest on August 15. After a $63 \%$ reduction, $p$-cymene content rose again to a higher value on October 15 . While the content of thymol was highest on April 15, it then decreased with the plant growth. Similarly, the content of neryl acetate reached the highest value on April 15 and had the second highest content on July 15, which was about $90 \%$ of the highest value. Only thymol could be detected in roots from June 1 to August 15, and the content was the highest on June 1.

In summary, the contents of four constituents in different parts of Eupatorium fortunei Turcz. reached the highest value in early July, followed by mid-September. These two time points could be considered as the best time to harvest the plant.

To the best of our knowledge, there are no studies regarding the relationship between the growth stages of Eupatorium fortunei Turcz. and the content of essential oil extracted from it. In other studies, it has been found that the contents of many biological active compounds in plants depend on growth time; the yields of compounds usually reach the highest at summer or flowering stages (July or August) [18, 19]. 


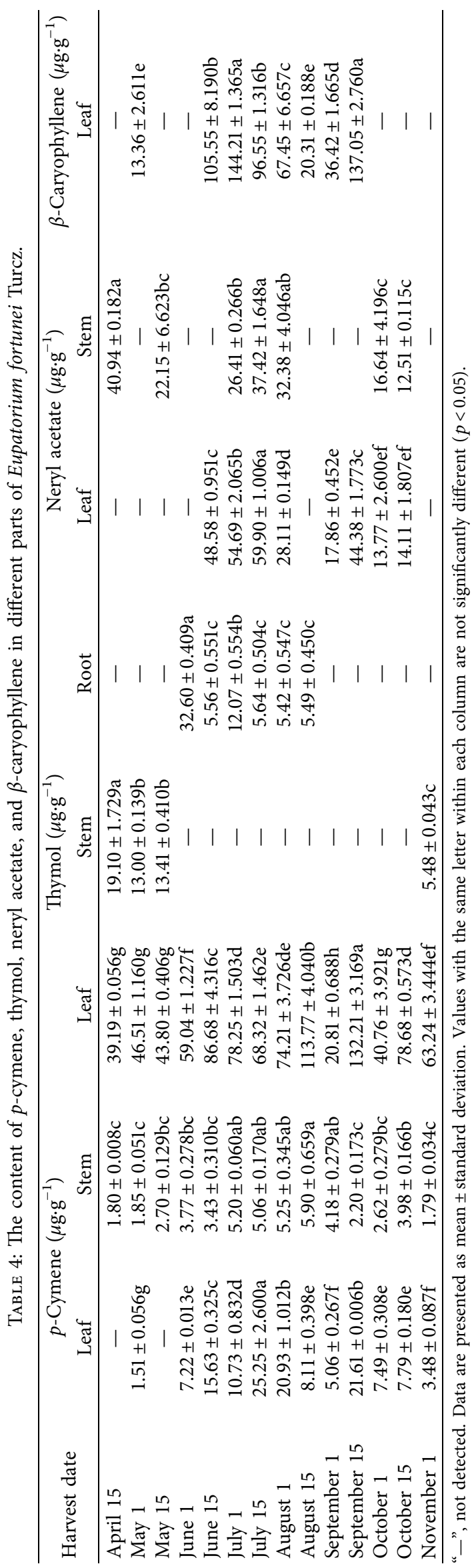




\section{Conclusions}

A quantitative determination method was developed and validated to assay the contents of $p$-cymene, thymol, neryl acetate, and $\beta$-caryophyllene in Eupatorium fortunei Turcz. by GC-MS/MS. The contents of the four constituents in samples from different parts were assayed and distribution was analyzed. All constituents could be detected in leaves. The contents order of constituents in different parts was leaves $>$ stems $>$ roots. From that, we could deduce that leaves should be the best medicinal part of Eupatorium fortunei Turcz. The growth stages of the plant strongly influence the content of four analytes in Eupatorium fortunei Turcz. The four constituents accumulated to the highest value in early July followed by mid-September. Accordingly, the best harvest time of Eupatorium fortunei Turcz. is early July and mid-September.

\section{Data Availability}

The data used to support the findings of this study are included within the article.

\section{Conflicts of Interest}

The authors declare that they have no conflicts of interest.

\section{Authors' Contributions}

Guanjun Nan and Lina Zhang contributed equally to this work.

\section{Acknowledgments}

This research was funded by the Key Research and Development Projects in Shaanxi Province, China (2020SF-319 and 2020ZDLSF05-12) and the National Natural Science Foundation of China (81373368).

\section{References}

[1] Jiangsu College of New Medicine, A Dictionary of the Traditional Chinese Medicines, People Hygiene Publisher, Shanghai, China, 1977.

[2] T. N. Pham, H. D. Pham, D. K. Dang et al., "Anticyanobacterial phenolic constituents from the aerial parts of Eupatorium fortunei Turcz." Natural Product Research, vol. 33, no. 9, pp. 1345-1348, 2019.

[3] J. Shi, M. Yuan, Y. Yu, S. B. Shi, and Y. G. Liu, "Chiral resolution, absolute configuration of two pairs of unusual monoterpene enantiomers from Eupatorium fortune," Tetrahedron Letters, vol. 61, no. 13, Article ID 151655, 2020.

[4] Chinese Pharmacopoeia Commission, Pharmacopoeia of the People's Republic of China, Medical Science and Technology Press, Beijing, China, 2020.

[5] P. Y. Liu, D. Liu, W. H. Li et al., "Chemical constituents of plants from the genus eupatorium (1904-2014)," Chemistry \& Biodiversity, vol. 12, no. 10, pp. 1481-1515, 2015.
[6] X. H. Wu and M. Zhong, "GC-MS analysis of chemical compositions of essential oils of Eupatorium fortunei extracted by $\mathrm{CO}_{2}$-supercritical-fluid-extraction or steam distillation extraction," China Pharmacy, vol. 20, no. 24, pp. 1888-1890, 2009.

[7] J. Liu and Y. Jin, "Extraction, GC-MS analysis and antibacterial activities of volatile oils from Eupatorium fortune," Journal of Hebei Agricultural Sciences, vol. 15, no. 3, pp. 150-154, 2011.

[8] X. T. Liang and G. L. Li, "The essential oil of Eupatorium fortunei Turcz." Acta Pharmaceutica Sinica, vol. 7, no. 4, pp. 131-135, 1959.

[9] D. G. Cai, Y. Z. Wang, and Y. Q. Lu, "Study on the active constituents of Eupatorium fortunei Turcz. in dispelling phlegm," China Journal of Chinese Materia Medica, vol. 8, no. 6, pp. 30-31, 1983.

[10] A. Kim, M. Im, N. H. Yim, and J. Y. Ma, "Reduction of metastatic and angiogenic potency of malignant cancer by Eupatorium fortunei via suppression of MMP-9 activity and VEGF production," Scientific Reports, vol. 4, no. 1, pp. 6994-7010, 2014.

[11] L. Liu, Q. Liu, and N. Y. Liu, "GC-MS analysis of volatile components of herba Eupatorii and herba Lycopi," Chinese Journal of Experimental Traditional Medical Formulae, vol. 14, no. 5, pp. 17-19, 2008.

[12] D. J. Liu, X. H. Zhao, J. Xue, S. L. Chen, and Q. Y. Bian, "Determination of caryophyllene in Eupatorium fortune," China Journal of Chinese Materia Medica, vol. 34, no. 22, pp. 2907-2909, 2009.

[13] M. Tori, Y. Ohara, K. Nakashima, and M. Sono, "Thymol derivatives from Eupatorium fortunei," Journal of Natural Products, vol. 64, no. 8, pp. 1048-1051, 2001.

[14] F. M. Zhu, B. Du, G. Xin, and C. J. Liu, "Analysis of chemical compositions of Eupatorium fortunei Turcz. essential oils," Food Science, vol. 29, no. 7, pp. 389-391, 2008.

[15] Y. Wang, J. Li, H. Wang et al., "Thymol derivatives from Eupatorium fortunei and their inhibitory activities on LPSinduced NO production," Phytochemistry Letters, vol. 7, pp. 190-193, 2014.

[16] Y. N. Tai, F. X. Zou, Q. R. Zhang et al., "Quantitative analysis of eight triterpenoids and two sesquiterpenoids in rhizoma alismatis by using UPLC-ESI/APCI-MS/MS and its application to optimisation of best harvest time and crude processing temperature," Journal of Analytical Methods in Chemistry, vol. 2019, Article ID 8320171, 2019.

[17] J. Hou, L. Liang, and Y. Wang, "Volatile composition changes in navel orange at different growth stages by HS-SPME-GCMS," Food Research International (Ottawa, Ont.), vol. 136, pp. 109333-109338, 2020.

[18] W. H. Liang, T. W. Chang, and Y. C. Chang, "Influence of harvest stage on the pharmacological effect of Angelica dahurica," Botanical Studies, vol. 59, no. 1, p. 14, 2018.

[19] I. Szymborska-Sandhu, J. L. Przybył, O. Kosakowska, K. Bączek, and Z. Węglarz, "Chemical diversity of Bastard Balm (Melittis melisophyllum L.) as affected by plant development," Molecules, vol. 25, no. 10, p. 2421, 2020.

[20] S. Kesen, "Characterization of aroma and aroma-active compounds of Turkish turmeric (Curcuma longa) extract," Journal of Raw Materials to Processed Foods, vol. 1, pp. 13-21, 2020. 
[21] H. Y. Zeng, "Fingerprint chromatogram of the volatile oils from Eupatorium fortunei with different methods," Food Science, vol. 25, no. 1, pp. 123-126, 2004.

[22] M. Xue, J. J. Qiao, Q. Wang et al., "Effects of different drying methods on quality and antibacterial activity of essential oils from Eupatorium fortune," Chinese Traditional Patent Medicine, vol. 40, pp. 2249-2254, 2018.

[23] F. Maggi, F. Papa, and S. Vittori, "Gas chromatography for the characterization of the mushroom-like flavor in Melittis melissophyllum L. (Lamiaceae)," Journal of Essential Oil Research, vol. 24, no. 4, pp. 321-337, 2012. 